UT-02-36

hep-th/0206010

June, 2002

\title{
Modular Invariance of Strings on PP-Waves with RR-flux
}

\author{
Tadashi Takayanagif \\ Department of Physics, Faculty of Science, University of Tokyo \\ Hongo 7-3-1, Bunkyo-ku, Tokyo, 113-0033, Japan
}

\begin{abstract}
We study the modular invariance of strings on pp-waves with RR-flux. We explicitly show that the one-loop partition functions of the maximally supersymmetric pp-waves and their orbifolds can be modular invariant in spite of the mass terms in the light-cone gauge. From this viewpoint, we also determine the spectrum of type $0 \mathrm{~B}$ theory on pp-wave and discuss its gauge theory dual. Furthermore, we investigate the spectrum of a non-supersymmetric orbifold and point out its supersymmetry enhancement in the Penrose limit.
\end{abstract}

\footnotetext{
${ }^{1}$ takayana@hep-th.phys.s.u-tokyo.ac.jp
} 


\section{Introduction}

Recently, much progress in understanding of the superstring theory in RR background has been made originating from the exactly solvable plane-wave background with RR-flux [1]. Interestingly, this background possesses an interpretation as the Penrose limit [2] of the near horizon geometry of D3-branes [3, 田. The near horizon limit of $N$ D3-branes is given by $A d S_{5} \times S^{5}$ and its metric is written as

$$
d s^{2}=R^{2}\left(-d t^{2} \cosh ^{2} \rho+d \rho^{2}+\sinh ^{2} \rho d \Omega_{3}^{2}+d \psi^{2} \cos ^{2} \theta+d \theta^{2}+\sin ^{2} \theta d \Omega_{3}^{\prime 2}\right),
$$

where the radius is given by $R=\left(4 \pi g_{s} N \alpha^{\prime 2}\right)^{\frac{1}{4}}$. After we take the Penrose limit $R \rightarrow \infty$ scaling as $x^{+}=(t+\psi) / 2 \mu, x^{-}=R^{2} \mu(t-\psi), \rho \rightarrow \rho / R, \theta \rightarrow \theta / R$, we obtain the maximally pp-wave metric [3, 团 (with the constant RR-flux $F_{+1234}=F_{+5678}=\mu$ )

$$
d s^{2}=-2 d x^{+} d x^{-}-\mu^{2} \sum_{i=1}^{8}\left(x^{i}\right)^{2}\left(d x^{+}\right)^{2}+\sum_{i=1}^{8}\left(d x^{i}\right)^{2} .
$$

Since we consider the RR background, we would like to quantize the world-sheet theory in the Green-Schwarz formulation of superstring [5]. Remarkably, the simple form of the metric (1.2) enables us to solve the world-sheet theory exactly in the light-cone gauge [1]. Moreover, the exact string spectrum on pp-waves has been successfully compared to the dual $\mathcal{N}=4$ gauge theory operators in [4]. Further studies of string and Mtheory in various pp-wave backgrounds and their holographically dual relations have been intensively carried out [6-30]2].

However, the light-cone gauge theory has an important fault that the conformal in-

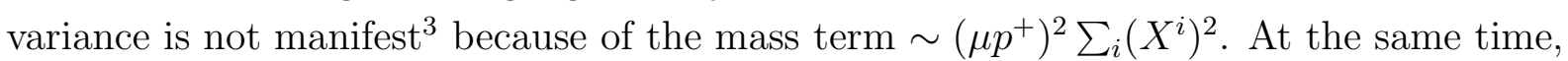
the modular invariance, which is crucial for the quantum consistency of any string theory, is also not clear. Thus we would like to investigate the modular invariance of string theory on pp-waves in this paper. An earlier discussion of modular transformation of open-string amplitudes into boundary state amplitudes has been found in [27]. Below we will calculate one-loop vacuum amplitudes of closed strings on pp-waves and examine their modular invariance. Since the pp-wave solution is the only solvable background with

\footnotetext{
${ }^{2}$ In these developments the authors have discussed the supersymmetry enhancement [6, 0, 23], orbifolded pp-waves [6, 11, 12, 13, 14, 22], the less supersymmetric pp-waves $18,15,16$, the algebras in the Penrose limit [3, 9, 29], D-branes in pp-waves [10, 23, 27], light-cone string field theory [21], holographic properties [18, 25], string interactions in the gauge theory side [25, 26], the Penrose limit in NSNS background [8, 23, 24, 29], another useful limit with a large value of spin [20, Matrix models [28] and some other gravitational properties 17 .

${ }^{3}$ The covariant string formalism of pp-wave background has been proposed in [19] and the conformal invariance has been checked.
} 
RR-flux at present, the study of modular invariance will also be very important to know properties and consistencies of string spectra in general RR backgrounds.

The modular invariance also gives us a good principle to determine string spectra in new string backgrounds. As we will see below, we can determine the spectrum of various orbifolds of pp-waves from this viewpoint. The examples discussed in this paper include pp-wave in type 0B theory, the non-supersymmetric oribifold $\mathbf{C} / \mathbf{Z}_{M}$ on pp-waves as well as the supersymmetric oribifold $\mathbf{C}^{2} / \mathbf{Z}_{M}$ theory on pp-waves [6, 11, 12, 13, 14]. We also discuss the non-supersymmetric analog of the duality relation $[4$ between the strings on pp-waves and the gauge theories in the examples of type $0 \mathrm{~B}$ and $\mathbf{C} / \mathbf{Z}_{M}$ pp-waves.

This paper is organized as follows. In section 2 , after a short review of world-sheet theory of strings on pp-waves, we calculate the one-loop vacuum amplitudes and examine their modular invariance. We apply this method to type 0 theory and determine the spectrum of the type 0B pp-wave model. We also discuss the dual gauge theory in this model. In section 3, we construct modular invariant partition function in both supersymmetric and non-supersymmetric orbifolded pp-waves. We also discuss the gauge theory dual of a non-supersymmetric orbifold theory and its supersymmetry enhancement. In section 4, we draw conclusions and present some discussions.

\section{Modular Invariance of Strings on PP-waves}

Here we would like to investigate modular properties of string theory on pp-waves. As we will see, we can prove its modular invariance performing a kind of a deformation of ordinary arguments in string theory with no RR-flux. See [27 for the analysis of modular transformation of open string amplitude into closed string one (Cardy's condition), which is useful for the discussions below.

First we present a brief review of the Green-Schwarz string on pp-waves [1]. We mainly follow the convention of [5, 8]. The coordinate of world-sheet is represented by $\tau$ and $\sigma \quad(0 \leq \sigma \leq \pi)$. After we take the light-cone gauge $X^{+}=2 \alpha^{\prime} p^{+} \tau, \gamma^{+} \theta^{1,2}=0$, there are eight bosonic fields $X^{i} \quad(i=1 \sim 8)$ and sixteen fermionic fields $S^{a}, \tilde{S}^{a} \quad(a=1 \sim 8)$. They are massive fields with the same mass and the world-sheet action becomes

$$
\begin{aligned}
S= & \frac{1}{\pi \alpha^{\prime}} \int d \tau d \sigma\left[\partial_{+} X^{i} \partial_{-} X^{i}-\left(\mu \alpha^{\prime} p^{+}\right)^{2}\left(X^{i}\right)^{2}\right] \\
& +\frac{i}{\pi} \int d \tau d \sigma\left[S^{a} \partial_{+} S^{a}+\tilde{S}^{a} \partial_{-} \tilde{S}^{a}-\left(2 \mu \alpha^{\prime} p^{+}\right) S^{a} \Pi^{a b} \tilde{S}^{b}\right]
\end{aligned}
$$

where we defined $\partial_{ \pm}=1 / 2\left(\partial_{\tau} \pm \partial_{\sigma}\right)$ and $\Pi=\gamma^{1} \gamma^{2} \gamma^{3} \gamma^{4}$.

The mode expansions of the bosonic fields which satisfy the equation of motion is 
given by

$$
X^{i}=\cos \left(2 \mu \alpha^{\prime} p^{+} \tau\right) x_{0}^{i}+\frac{\sin \left(2 \mu \alpha^{\prime} p^{+} \tau\right)}{\mu p^{+}} p_{0}^{i}+i \sqrt{\frac{\alpha^{\prime}}{2}} \sum_{n \neq 0}\left(\frac{\alpha_{n}^{i}}{\omega_{n}} e^{-2 i \omega_{n} \tau-2 i n \sigma}+\frac{\tilde{\alpha}_{n}^{i}}{\omega_{n}} e^{-2 i \omega_{n} \tau+2 i n \sigma}\right),
$$

where we have defined

$$
\omega_{n}=\frac{n}{|n|} \sqrt{n^{2}+\left(\mu \alpha^{\prime} p^{+}\right)^{2}} .
$$

The canonical quantization imposes the following commutation relations

$$
\left[\alpha_{n}^{i}, \alpha_{m}^{i}\right]=\left[\tilde{\alpha}_{n}^{i}, \tilde{\alpha}_{m}^{i}\right]=\omega_{n} \delta_{n+m, 0} \delta^{i j}, \quad\left[\alpha_{0}^{i}, \alpha_{0}^{\dagger i}\right]=\omega_{0},
$$

where we redefined the zero-modes as $\alpha_{0}^{i}=\sqrt{\alpha^{\prime} / 2}\left(p_{0}^{i}-i \mu p^{+} x_{0}^{i}\right), \alpha_{0}^{\dagger i}=\sqrt{\alpha^{\prime} / 2}\left(p_{0}^{i}+i \mu p^{+} x_{0}^{i}\right)$.

We can also perform the mode expansions of fermions $S^{a}$ and $\tilde{S^{a}}$ similarly. We denote their oscillators as $S_{n}^{a}$ and $\tilde{S}_{n}^{a}$ and (the linear combination of) the zero-modes as $S_{0}^{a}$ and $S_{0}^{\dagger a}$ such that the following anti-commutation relations are satisfied

$$
\left\{S_{n}^{a}, S_{m}^{b}\right\}=\left\{\tilde{S}_{n}^{a}, \tilde{S}_{m}^{b}\right\}=\delta_{n+m, 0} \delta^{a b}, \quad\left\{S_{0}^{a}, S_{0}^{\dagger a}\right\}=\delta^{a b} .
$$

Then we find the spectrum in the string theory on pp-wave as follows. The light-cone Hamiltonian $\mathcal{H}=-p^{-}$is given by

$$
\begin{aligned}
\mathcal{H} & =\frac{1}{\alpha^{\prime} p^{+}}\left(\alpha_{0}^{\dagger i} \alpha_{0}^{i}+\omega_{0} S_{0}^{\dagger a} S_{0}^{a}+\sum_{n=1}^{\infty}\left(\alpha_{-n}^{i} \alpha_{n}^{i}+\tilde{\alpha}_{-n}^{i} \tilde{\alpha}_{n}^{i}+\omega_{n} S_{-n}^{a} S_{n}^{a}+\omega_{n} \tilde{S}_{-n}^{a} \tilde{S}_{n}^{a}\right)\right) \\
& \equiv \sum_{n=-\infty}^{\infty} N_{n} \sqrt{\mu^{2}+\left(\frac{n}{\alpha^{\prime} p^{+}}\right)^{2}} .
\end{aligned}
$$

The vacuum state $|0\rangle$ has the zero light-cone energy $H|0\rangle=0$ because it is defined such that it is annihilated by the operators $\alpha_{-n}^{i}, \tilde{\alpha}_{-n}^{i}, S_{-n}^{a}, \tilde{S}_{-n}^{a}(n>0)$ and $\alpha_{0}^{i}$ and $S_{0}^{a}$. The level matching condition is also written as

$$
\mathcal{P}=\sum_{n=-\infty}^{\infty} n N_{n}=0
$$

Now the one-loop vacuum amplitude (partition function) can be defined as follows

$$
Z=c \int \frac{d \tau d \bar{\tau}}{\tau_{2}} \int d p^{+} d p^{-} \operatorname{Tr}\left[e^{-2 \pi \alpha^{\prime} \tau_{2} p^{+}\left(p^{-}+\mathcal{H}\right)+2 \pi i \tau_{1} \mathcal{P}}\right]
$$

where Tr denotes the trace in the Hilbert space of the light-cone string theory'; $c$ is a constant factor, which we will neglect below; $\tau=\tau_{1}+i \tau_{2}$ denotes the moduli of two dimensional torus as usual. The modular invariance of the partition function enables us to restrict the integration of the torus moduli $\tau, \bar{\tau}$ to the fundamental region.

\footnotetext{
${ }^{4}$ For earlier discussions of closed string partition function see 86. Note that here we define the trace with the minus sign for all spacetime fermions so that the amplitude represents the vacuum energy.
} 


\subsection{Modular Invariance of Type II String on PP-wave}

Before we compute the vacuum amplitude, it is useful to define the following partition function $Z_{a, b}^{(m)}(\tau, \bar{\tau})$, which has a nice modular property,

$$
\begin{gathered}
Z_{a, b}^{(m)}(\tau, \bar{\tau})=e^{4 \pi \tau_{2} \Delta_{b}^{(m)}} \prod_{n=-\infty}^{\infty}\left(1-e^{-2 \pi \tau_{2} \sqrt{m^{2}+(n+b)^{2}}+2 \pi i \tau_{1}(n+b)+2 \pi i a}\right) \\
\times\left(1-e^{-2 \pi \tau_{2} \sqrt{m^{2}+(n-b)^{2}}+2 \pi i \tau_{1}(n-b)-2 \pi i a}\right) .
\end{gathered}
$$

The factor $\Delta_{b}^{(m)}$ corresponds to the zero-energy (Casimir energy) of a 2D complex scalar boson $\phi$ of mass $m$ with the twisted boundary condition $\phi(\tau, \sigma+\pi)=e^{2 \pi i b} \phi(\tau, \sigma)$ and its explicit form is defined by

$$
\Delta_{b}^{(m)}=-\frac{1}{2 \pi^{2}} \sum_{p=1}^{\infty} \int_{0}^{\infty} d s e^{-p^{2} s-\frac{\pi^{2} m^{2}}{s}} \cos (2 \pi b p) .
$$

In the massless limit this zero energy correctly reproduces the familiar value

$$
\lim _{m \rightarrow 0} \Delta_{b}^{(m)}=\frac{1}{24}-\frac{1}{8}(2 b-1)^{2} .
$$

The modular property of the above defined function is given by

$$
\begin{aligned}
& Z_{a, b}^{(m|\tau|)}\left(-\frac{1}{\tau},-\frac{1}{\bar{\tau}}\right)=Z_{-b, a}^{(m)}(\tau, \bar{\tau}), \\
& Z_{a, b}^{(m)}(\tau+1, \bar{\tau}+1)=Z_{a+b, b}^{(m)}(\tau, \bar{\tau}) .
\end{aligned}
$$

The latter identity is easy to check and the more nontrivial relation (the former one) can be proved by using the Poisson resummation formula as shown in the appendix. The important point is that we must shift the value of mass parameter as $m \rightarrow m|\tau|$. This is natural because the theory is massive and thus is not conformal invariant. Note also that since we consider the two dimensional massive theory, there is not any definite distinction between left-moving and right-moving sector. Thus we cannot define a sort of a chiral partition as in massless case. It also enjoys other useful properties

$$
Z_{a+1, b}^{(m)}(\tau, \bar{\tau})=Z_{a, b+1}^{(m)}(\tau, \bar{\tau})=Z_{a, b}^{(m)}(\tau, \bar{\tau}), \quad Z_{-a,-b}^{(m)}(\tau, \bar{\tau})=Z_{a, b}^{(m)}(\tau, \bar{\tau})
$$

In the conformal limit, $m \rightarrow 0$ it is reduced to the familiar theta function

$$
\lim _{m \rightarrow 0} Z_{a, b}^{(m)}(\tau, \bar{\tau}) \frac{\sqrt{Z_{0,0}^{(m)}(\tau, \bar{\tau})}}{2 \pi \tau_{2} m}=e^{-2 \pi b^{2} \tau_{2}}\left|\theta_{1}(a+b \tau \mid \tau)\right|^{2} .
$$

5 In ref.27 the authors defined the real partition functions $f_{i}^{(m)}(q), \quad(i=1,2,3,4)$. Our complex partition function $Z_{a, b}^{(m)}(\tau, \bar{\tau})$ includes (the square of) them as the particular cases $\tau_{2}=0$ and $(a, b)=$ $(0,0),(0,1 / 2),(1 / 2,0),(1 / 2,1 / 2)$. 
Now let us move on to the investigation of the one-loop string partition function on the maximally supersymmetric pp-wave. Its explicit form is given by

$$
Z_{\mathrm{IIB}}=\int \frac{d \tau d \bar{\tau}}{\tau_{2}} \int d p^{+} d p^{-} e^{-2 \pi \alpha^{\prime} \tau_{2} p^{+} p^{-}}\left(\frac{Z_{0,0}^{\left(\alpha^{\prime} \mu p^{+}\right)}(\tau, \bar{\tau})}{Z_{0,0}^{\left(\alpha^{\prime} \mu p^{+}\right)}(\tau, \bar{\tau})}\right)^{4}
$$

Note that the denominator and numerator of the modular function are the same and thus the quotient is given by not zero but one?. This phenomenon is due to the spacetime supersymmetry of the pp-wave background. Since the dynamical supercharges $Q^{-}$commute with $\mathcal{H}$ (see [1]), we can regard the total partition function as the Witten index of $Q^{-}$. Thus only the (bosonic) ground state $|0\rangle$ can contribute to it and we obtain the unit values.

The modular invariance of (2.16) does hold formally without using this fact, that is, independently with respect to bosonic and fermionic contribution, though the integrations of $p^{+}$and $p^{-}$diverge. This is shown by noting the modular invariant combination $d \tau d \bar{\tau} / \tau_{2}^{2}$ and performing the rescaling

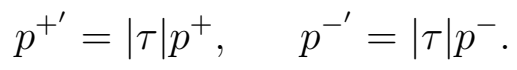

After we perform an analytic continuation of the momentum integration and integrate the torus moduli $\tau, \bar{\tau}$ in the fundamental region, we obtain a finite value of the amplitude.

\subsection{Type 0 String on PP-wave}

Since in the previous example of the maximally supersymmetric pp-wave in type IIB theory the modular function part is rather trivial due to the Bose-Fermi degeneracy, next we would like to consider a more non-trivial case of type $0 \mathrm{~B}$ pp-wave'?

In type 0 string theory there are two types of D-branes each called electric and magnetic D-branes [31] (for a review of type 0 theory see [32]). Since the near horizon limit of $N$ electric D3-branes and $N$ magnetic D3-branes in type 0B theory is given by $A d S_{5} \times S^{5}$

\footnotetext{
${ }^{6}$ If we take the limit $\mu=0$, one may think that the partition function should vanish due to Jacobi identity. This does not contradict our result (2.16) because our function $\sim 1 / Z_{0,0}^{(m) 4}$ includes the divergent volume factor.

${ }^{7}$ After we submitted this paper, we were informed by A.A.Tseytlin of another definition of one-loop vacuum amplitude based on path-integral calculations, which seems to be zero. The author is very grateful to A.A.Tseytlin for sending his unpublished notes and our correspondence. In this paper we only discuss the partition function defined in the operator formalism.

8 The author would like to thank T. Eguchi and Y.Sugawara very much for related comments on the property of partition function.

${ }^{9}$ While preparing this paper for publication, there appears the partially overlapped paper [30] which discusses string on type $0 \mathrm{~B}$ pp-wave and duality to gauge theory independently.
} 
[31], the pp-wave background can be realized by considering the Penrose limit of this background. The gauge theory on these branes is non-supersymmetric and its gauge group is given by $S U(N) \times S U(N)$. Generalizing the arguments in the ordinary type 0 theory with no RR-flux [31], we argue that the spectrum can be obtained by twisting that of type IIB pp-wave by the $\mathbf{Z}_{2}$ projection $(-1)^{F_{S}}$, where $F_{S}$ means the spacetime fermion number. Then we find the modular invariant partition function, which is more complicated than in the type IIB case

$$
\begin{aligned}
Z_{0 \mathrm{~B}}= & \int \frac{d \tau d \bar{\tau}}{\tau_{2}} \int d p^{+} d p^{-} e^{-2 \pi \alpha^{\prime} \tau p_{2} p^{+} p^{-}} \\
& \times \frac{Z_{0,0}^{\left(\alpha^{\prime} \mu p^{+}\right)}(\tau, \bar{\tau})^{4}+Z_{1 / 2,0}^{\left(\alpha^{\prime} \mu p^{+}\right)}(\tau, \bar{\tau})^{4}+Z_{0,1 / 2}^{\left(\alpha^{\prime} \mu p^{+}\right)}(\tau, \bar{\tau})^{4}+Z_{1 / 2,1 / 2}^{\left(\alpha^{\prime} \mu p^{+}\right)}(\tau, \bar{\tau})^{4}}{2 Z_{0,0}^{\left(\alpha^{\prime} \mu p^{+}\right)}(\tau, \bar{\tau})^{4}} .
\end{aligned}
$$

The first two functions correspond to the untwisted sector of the $\mathbf{Z}_{2}$ twist and the last two correspond to the twisted sector with the shifted modings as $\alpha_{n+1 / 2}^{i}, S_{n+1 / 2}^{a}$. We have determined their non-trivial zero energy by requiring the modular invariance. Note also that the spectrum is purely bosonic as in the flat background.

Next let us briefly discuss the duality of this pp-wave background to the gauge theory. This will give a non-supersymmetric generalization of the result in [4]. To see this it is useful to note that our system in type 0 theory can be obtained from a system of $2 N$ D3-branes in type IIB theory $\left(S U(2 N)\right.$ gauge theory) by the projection $(-1)^{F_{S}}$. After the projection, open strings between the same kind of D-branes are bosonic and open strings between the different kind of D-branes (electric and magnetic) are fermionic. More explicitly, the $\mathbf{Z}_{2}$ projection acts as $\mathbb{U}^{\mathbb{T}}(-1)^{F_{S}} \cdot \sigma_{3} \otimes \mathbf{1}_{N}$ on the open string spectrum with $2 N \times 2 N$ Chan-Paton matrices [31]. Then we can see the duality to the gauge theory by applying the arguments in orbifold theory [11, 12, 13]. The ground state of untwisted sector corresponds to the operator $\operatorname{Tr}\left(Z^{J}\right)$, where the trace is defined for $2 N \times 2 N$ matrices. The field $Z=\phi_{5}+i \phi_{6}$ denotes a (complex) transverse scalar in the gauge theory and has the $U(1)$ charge $J=1$. The twisted sector ground state is dual to the operator $\operatorname{Tr}\left(\sigma_{3} Z^{J}\right)$. The excitations of these vacuum states correspond to the insertions of the covariant derivatives $D_{i}(i=1 \sim 4)$ or the other transverse scalars $\phi^{i} \quad(i=1 \sim 4)$ taking the summation over their possible positions with nontrivial phase factors in the same way as in [4]. Fermions $\chi^{a} \quad(i=1 \sim 8)$, which have the $U(1)$ charge $J=1 / 2$, can also be treated similarly. For example, in the twisted sector we obtain the correspondence

$$
S_{-n-1 / 2}^{a} \tilde{S}_{-n-1 / 2}^{b}|0\rangle \leftrightarrow \sum_{l=0}^{J} e^{\frac{2 \pi i l(n+1 / 2)}{J}} \operatorname{Tr}\left[\sigma_{3} Z^{l} \chi^{a} Z^{J-l} \chi^{b}\right],
$$

\footnotetext{
${ }^{10}$ Here $\sigma_{3}$ is the Pauli matrix. Below we omit the trivial part $\mathbf{1}_{N}$.
} 
where the twisted boundary condition corresponds to the shift of the 'momentum' $n$ by $1 / 2$ because of the non-trivial commutation relation $\chi^{a} \sigma_{3}=-\sigma_{3} \chi^{a}$ in the gauge theory as already discussed in the context of orbifolded pp-waves [12, 13]. Interestingly, the spacetime fermions of type 0 string theory, which exist only in open string sectors, just correspond to the purely bosonic closed string spectrum via the holography. If we view this in an opposite way, the absence of spacetime fermions in the closed string theory is consistent with the fact that the trace does vanish when we insert the fermions into $\operatorname{Tr}\left(Z^{J}\right)$ or $\operatorname{Tr}\left(\sigma_{3} Z^{J}\right)$ odd times. It will also be interesting to perform gauge theory calculations and see the matching of spectrum in detail including the non-trivial zero-energy in the twisted sector (see (2.20) and later discussions). We would like to leave further analyses for future problems.

Finally we would like to mention the closed string tachyon in type 0 theory. As is well-known, there is a closed string tachyon in the flat background of type 0 string. In our case of pp-waves we can detect the tachyonic excitation by examining the divergent factor in the partition function i.e. $\sim \int d \tau_{2} e^{\tau_{2} \beta} \quad(\beta>0)$. Thus we have only to know the difference of zero-energy for the latter two functions in (2.18)

$$
\Delta_{1 / 2}^{\left(\mu \alpha^{\prime} p^{+}\right)}-\Delta_{0}^{\left(\mu \alpha^{\prime} p^{+}\right)}=\frac{1}{\pi^{2}} \sum_{p=0}^{\infty} \int_{0}^{\infty} d s e^{-(2 p+1)^{2} s-\frac{\left(\pi \alpha^{\prime} \mu p^{+}\right)^{2}}{s}}>0
$$

This shows that we have always a tachyon for finite values of $\mu$. If we set $\mu=0$, then the value becomes $\Delta_{1 / 2}^{(0)}-\Delta_{0}^{(0)}=1 / 8$ and the known tachyon mass $m^{2}=-\frac{2}{\alpha^{\prime}}$ is reproduced. More interestingly, if we consider the infinite value of $\mu$, then the tachyon seems to disappear.

\section{Modular Invariance of Orbifolded PP-Waves}

The Green-Schwarz string on pp-wave background is originally constructed by employing its supersymmetries in the superspace formalism [1]. Thus it does not seem to be easy to obtain the less supersymmetric models in the Green-Schwarz formalism. However, the orbifold procedure allows us to realize such models with few difficulties' We have already discussed the non-supersymmetric pp-wave in type 0 theory as a (non-geometric) orbifold and thus here we would like to investigate the orbifold defined by geometric projections.

The supersymmetric orbifolded pp-waves has been considered and its duality to the quiver gauge theory [33] has been checked including twisted sectors [11, 12, 13]. In these

\footnotetext{
${ }^{11}$ We can also consider the Penrose limit of $A d S_{3} \times S^{3} \times \mathrm{M}_{4}$ system [4, 8] as a less supersymmetric example, where the four dimensional manifold $\mathrm{M}_{4}$ represents $c=6$ conformal field theory without RRflux. The modular invariance of its partition function can be shown in the same way.
} 
arguments the authors impose the twisted boundary conditions just as in the ordinary well-studied cases of orbifolds with no RR-flux. Since the twisted sectors are originally added to physical sectors in order to keep modular invariance (see e.g. [32]), it is very important to prove this property for orbifolded pp-waves.

\section{Supersymmetric Orbifold $\mathbf{C}^{\mathbf{2}} / \mathbf{Z}_{M}$}

Let us first discuss the orbifolded pp-waves which is equivalent to the Penrose limit of the near horizon geometry $A d S_{5} \times S^{5} / \mathbf{Z}_{M}$ of $N M$ D3-branes at the orbifold $\mathbf{C}^{2} / \mathbf{Z}_{M}$. The orbifold action is given by

$$
X=X^{4}+i X^{5} \mapsto e^{2 \pi i / M} X, \quad Y=X^{6}+i X^{7} \mapsto e^{-2 \pi i / M} Y,
$$

and the others $X^{1}, X^{2}, X^{3}, X^{4}$ are unchanged because they are in the direction of $A d S_{5}$ (only the coordinates of $\Omega_{3}^{\prime}$ in (1.1) are orbifolded). The fermions $S^{a}, \tilde{S^{a}}$, which belong to the spinor representation, are also acted in the same way because there are half of maximal spacetime supersymmetries preserved. The partition function is written as follows

$$
Z_{\mathrm{orbs}}=\int \frac{d \tau d \bar{\tau}}{\tau_{2}} \int d p^{+} d p^{-} e^{-2 \pi \alpha^{\prime} \tau_{2} p^{+} p^{-}} \frac{1}{M} \sum_{k, l=0}^{M-1}\left(\frac{Z_{0,0}^{\left(\alpha^{\prime} \mu p^{+}\right)}(\tau, \bar{\tau})}{Z_{0,0}^{\left(\alpha^{\prime} \mu p^{+}\right)}(\tau, \bar{\tau})}\right)^{2}\left(\frac{Z_{k / M, l / M}^{\left(\alpha^{\prime} \mu p^{+}\right)}(\tau, \bar{\tau})}{Z_{k / M, l / M}^{\left(\alpha^{\prime} \mu p^{+}\right)}(\tau, \bar{\tau})}\right)^{2}
$$

where the summations over $k$ and $l$ represent the orbifold projection and the twisted sectors, respectively. We can show the modular invariance by using the property (2.12) only if we include the twisted sectors as in the above form (3.2). Since there exist the partial supersymmetries preserved in this orbifolded pp-wave, the Bose-Fermi degeneracy occurs and thus the quotient of the modular functions is equal to a constant. We would also like to comment that we can impose orbifold projections for all coordinates $X^{1} \sim X^{8}$ in the same way, which will be the Penrose limit of the orbifold not only with respect to $S^{5}$ but also to $A d S_{5}$.

\section{Non-Supersymmetric Orbifold $\mathbf{C} / \mathbf{Z}_{M}$}

It will be also interesting to consider the non-supersymmetric orbifold of the maximally supersymmetric pp-wave integer ${ }^{[3]}$ ) in pp-waves, where the orbifold projection is defined by

$$
X=X^{4}+i X^{5} \mapsto e^{2 \pi i L / M} X,
$$

\footnotetext{
${ }^{12}$ In the paper [13] the non-supersymmetric type of the orbifold $\mathbf{C}^{2} / \mathbf{Z}_{M}$ was considered. The discussion here can also be applied to this case without serious modifications.

${ }^{13}$ Note that if we assume $M$ even, then it should be identified with an orbifold in type 0 theory [34, 35.
} 
where $L$ is an even integer (see e.g. 36, 35] for earlier discussions of its partition function without RR-flux). Its modular invariant one-loop partition function is given by

$$
Z_{\text {orbn }}=\int \frac{d \tau d \bar{\tau}}{\tau_{2}} \int d p^{+} d p^{-} e^{-2 \pi \alpha^{\prime} \tau_{2} p^{+} p^{-}} \frac{1}{M} \sum_{k, l=0}^{M-1} \frac{\left(Z_{\frac{L k}{2 M}, \frac{L l}{2 M}}^{\left(\alpha^{\prime} \mu p^{+}\right)}(\tau, \bar{\tau})\right)^{4}}{\left(Z_{0,0}^{\left(\alpha^{\prime} \mu p^{+}\right)}(\tau, \bar{\tau})\right)^{3} Z_{\frac{L k}{M}, \frac{L l}{M}}^{\left(\alpha^{\prime} \mu p^{+}\right)}(\tau, \bar{\tau})}
$$

The closed string tachyons in this model can also be examined as in the type 0 pp-wave and we find 'localized tachyons' only in twisted sectors as in the ordinary orbifold [34. The duality to the non-supersymmetric quiver gauge theory can also be examined as in the supersymmetric orbifold $\mathbf{C}^{2} / \mathbf{Z}_{M}$ formally, though the matching of conformal dimension of operators is difficult to check due to the absence of BPS arguments. The $l$-th twisted sector corresponds to the insertion of the diagonal matrix

$$
\gamma_{l}=\operatorname{diag}\left(1, e^{\frac{2 \pi i L l}{M}}, e^{\frac{4 \pi i L l}{M}}, \cdots, e^{\frac{2 \pi i L(M-1) l}{M}}\right),
$$

into the trace $\operatorname{Tr}\left[Z^{J}\right]$. It would also be intriguing to find the gauge theoretic origin of the non-trivial zero-energy $\Delta_{l} \equiv 4 \Delta_{\frac{L l}{2 M}}^{\left(\alpha^{\prime} \mu p^{+}\right)}-3 \Delta_{0}^{\left(\alpha^{\prime} \mu p^{+}\right)}-\Delta_{\frac{L l}{M}}^{\left(\alpha^{\prime} \mu p^{+}\right)}>0$ in the $l$-th twisted sector.

\section{Supersymmetry Enhancement in Non-Supersymmetric Gauge Theory}

There is another Penrose limit of the previous non-supersymmetric orbifold. In the near horizon background (1.1) we can impose the $\mathbf{Z}_{M}$ projection as follows

$$
Z=R \cos \theta e^{i \psi} \mapsto e^{2 \pi i L / M} Z
$$

After taking the limit, this leads to the periodicity in the light-cone direction (setting $\mu=1)$

$$
\left(x^{+}, x^{-}\right) \sim\left(x^{+}+\frac{\pi L}{M}, x^{-}+\frac{2 \pi L R^{2}}{M}\right),
$$

where the radius is given by $R=\left(4 \pi N M g_{s} \alpha^{2}\right)^{\frac{1}{4}}$. When the $x^{+}$direction is compactified, the background breaks maximal supersymmetries because the Killing spinor depends (only) on $x^{+}$[3]. If we take ,however, the limit $M, N \rightarrow \infty$ keeping $L$ and $\frac{N}{M}$ finite, then we obtain the DLCQ compactification of maximally supersymmetric pp-waves (1.2)

$$
x^{-} \sim x^{-}+2 \pi R^{-} \quad\left(R^{-}=2 \alpha^{\prime} L \sqrt{\pi g_{s} N / M}\right) .
$$

This background preserves all of the 32 supersymmetries and thus the supersymmetry is enhanced in this limit even though the original background is non-supersymmetric ${ }^{\mathbb{t}}$. The

\footnotetext{
${ }^{14}$ The enhancement of supersymmetry in the Penrose limit of non-supersymmetric models with NSNSflux has also been discussed in [23].
} 
similar type of the appearance of the DLCQ compactification has already been discussed in 222 in the case of the supersymmetric orbifold $\mathbf{C}^{2} / \mathbf{Z}_{M}$.

Let us consider the holographic relation between the string states of the DLCQ and the operators in the non-supersymmetric quiver gauge theory at the classical level applying the arguments in [22]. To begin with, we obtain the following correspondence

$$
p^{-}=\Delta-J, \quad 2 p^{+}=\frac{\Delta+J}{R^{2}}
$$

where $\Delta$ and $J$ are the conformal dimension and $\mathrm{U}(1)$ charge defined below, respectively. The light-cone compactification leads to the quantized momentum $p^{+}=\frac{k}{R^{-}}, \quad(k \in \mathbf{Z})$. The light-cone Hamiltonian $\mathcal{H}\left(=-p^{-}\right)$remains the same as (2.6) and the level matching is given by $\sum_{n=-\infty}^{\infty} n N_{n}=k w$, where $w$ is the winding number in the $x^{-}$direction! $\square$.

Next we would like to examine the quiver gauge theory. There are six transverse scalars and we denote them by $\phi^{1}, \phi^{2}, \phi^{3}, \phi^{4}$ and $Z=\phi^{5}+i \phi^{6}\left(\bar{Z}=\phi^{5}-i \phi^{6}\right)$. The $\mathbf{Z}_{M}$ projection acts only on $Z$ and $\bar{Z}$ in the same as (3.3). The fields which have non-zero R-charges are the scalars $Z \quad(J=1), \bar{Z} \quad(J=-1)$ and fermions $\chi^{1} \sim \chi^{8} \quad(J=1 / 2)$, $\tilde{\chi}^{1} \sim \tilde{\chi}^{8} \quad(J=-1 / 2)$. We can expect that only the fields which have the value $\Delta-J=1$ will survive in the Penrose limit as in 4 . In order to be $\mathbf{Z}_{M}$ invariant the number of the insertions of $Z$ in any single trace operator should be a multiple of $M(=M k)$ and this just corresponds to the quantized light-cone momentum $k$ in the DLCQ compactification (3.8). Thus the vacuum state $|k, w=0\rangle$ of DLCQ string corresponds to the operator $\operatorname{Tr}\left[Z^{M k}\right]$, where the trace is defined for $N M \times N M$ matrices. Then the excited states can be obtained as the insertions of covariant derivatives $D_{i}$, scalar fields $\phi^{i}$, or fermions $\chi^{a}$. For example, we obtain the following translation for each insertion $(i=5,6,7,8)$

$$
\alpha_{-n}^{i} \leftrightarrow \phi^{i} \gamma_{n / k}, \quad \tilde{\alpha}_{-n}^{i} \leftrightarrow \phi^{i} \gamma_{-n / k}
$$

where the matrix $\gamma$ is defined in (3.5). Note that after taking the trace and summation over their positions $l$ (see (2.19)), the insertions of extra matrices $\gamma_{ \pm n / k}$ are equivalent to both the familiar phase factor $e^{ \pm \frac{2 \pi i n l}{J}}$ and the overall matrix $\prod_{n}\left(\gamma_{n / k}\right)^{N_{n}}=\gamma_{m}$ in the first position in the trace. The latter matrix comes from the orbifold action on Chan-Paton matrices in the same way as before (see the arguments below (2.19) or [12, 13]).

Even though the above arguments neglect quantum corrections, our 'too good' results implies that the result will not be changed substantially if we go beyond the classical analysis $\square$. We would also like to mention that the result suggests the possibility of

\footnotetext{
${ }^{15}$ The modular invariance of partition function in DLCQ theory seems to be subtle because the scaling (2.17) is not allowed. This problem may suggest the requirement of the detailed analysis of Lorentzian torus. We leave this as a future problem.

${ }^{16}$ Here we ignore the presence of closed string tachyon in twisted sectors. Notice that the tachyonic
} 
'deconstructing' supersymmetric five dimensional gauge theory or six dimensional $(2,0)$ theory from the non-supersymmetric gauge theory (cf. 37, 38, 22]).

\section{Conclusions and Discussions}

In this paper we have investigated the modular invariance of strings on pp-waves. At first sight, the models do not even seem to be conformal invariant because of the mass terms for the world-sheet fields in the light-cone gauge. However, the total theory including the light-cone directions $x^{+}, x^{-}$should be completely conformal invariant as examined in the covariant formalism [19]. Our results show that the modular invariance can also be satisfied formally, though the calculations include (perhaps inevitable) divergence due to Lorentz signature. Thus we have obtained an important quantum consistency of the string theory on pp-waves. It would be very interesting to see the interpretation of the modular invariance and the scaling (2.17) from the viewpoint of the dual gauge theory. The one-loop contribution will be important when we consider non-planar diagrams in the gauge theories.

Since the modular invariance puts a rather strict constraint in string theory, it is a useful guide when we construct a new theory. Based on this policy we have constructed the partition function of various orbifolded pp-waves. These include the supersymmetric orbifolds, non-supersymmetric type IIB orbifold and type 0B theory. In the latter two examples, we also gave a brief survey of the correspondence between the strings on ppwaves and dual (quiver) gauge theories. In particular, we pointed out that supersymmetry can be enhanced in the specific Penrose limit of the non-supersymmetric orbifold and the DLCQ compactification of pp-waves can be obtained in a similar way to [22]. We also find the possibility that in these examples the tachyonic instability may be reduced for large values of RR-flux parameter.

We believe the method developed in this paper will be useful in more general models. For example, we will be able to discuss the world-sheet properties of fractional D-branes [33 in orbifolded pp-waves and also the modular properties of compactified pp-waves [16. Our results may also give a helpful hint when one tries to know an unknown NS-R formulation of strings on pp-waves with RR-flux.

instability seems to be reduced when we assume a large value of $\mu$ in the same way as in type 0 theory (2.20). 


\section{Acknowledgments}

I am grateful to T.Eguchi and Y.Sugawara for useful comments. I also thank A.A.Tseytlin for sending his unpublished notes and our correspondence. The research of T.T. was supported in part by JSPS Research Fellowships for Young Scientists.

\section{A Detailed Calculations of Modular Transformations}

Here we show the (deformed) modular transformation of $Z_{a, b}^{(m)}(\tau, \bar{\tau})$. In the following calculations we will only use the Poisson resummation formula

$$
\sum_{n \in \mathbf{Z}} e^{-\pi a n^{2}+2 \pi i n b}=\frac{1}{\sqrt{a}} \sum_{\hat{n} \in \mathbf{Z}} e^{-\frac{\pi}{a}(\hat{n}-b)^{2}},
$$

and the identity

$$
\frac{1}{\sqrt{\pi}} \int_{0}^{\infty} d s s^{-\frac{1}{2}} e^{-s-\frac{t^{2}}{4 s}}=e^{-t}
$$

generalizing the earlier computations done in [27].

First we consider the logarithm of $Z_{a, b}^{(m)}(\tau, \bar{\tau})$ and perform the Poisson resummation

$$
\begin{aligned}
& \log Z_{a, b}^{(m)}(\tau, \bar{\tau})-4 \pi \tau_{2} \Delta_{b}^{(m)} \\
& =\sum_{n \in \mathbf{Z}}\left[\log \left(1-e^{-2 \pi \tau_{2} \sqrt{m^{2}+(n+b)^{2}}+2 \pi i \tau_{1}(n+b)+2 \pi i a}\right)+\log \left(1-e^{-2 \pi \tau_{2} \sqrt{m^{2}+(n-b)^{2}}+2 \pi i \tau_{1}(n-b)-2 \pi i a}\right)\right] \\
& =-\frac{1}{\sqrt{\pi}} \sum_{n \in \mathbf{Z}} \sum_{p=1}^{\infty} \int d s s^{-\frac{1}{2}}\left[e^{-p^{2} s-\pi^{2} \frac{\tau_{2}^{2}}{s}\left(m^{2}+(n+b)^{2}\right)+2 \pi i \tau_{1} p(n+b)+2 \pi i a p}+(\text { conjugate term) }]\right. \\
& =-\frac{1}{\pi \tau_{2}} \sum_{p=-\infty}^{\infty} \sum_{\hat{n}=1}^{\infty} \int_{0}^{\infty} d s\left[\exp \left(-\frac{|\tau|^{2}}{\tau_{2}^{2}} p^{2} s+2 \pi i a p-\frac{2 \tau_{1}}{\tau_{2}^{2}} \hat{n} p s-\frac{\pi^{2} \tau_{2}^{2} m^{2}}{s}-\frac{s \hat{n}^{2}}{\tau_{2}^{2}}-2 \pi i b \hat{n}\right)\right. \\
& +(\text { conjugate term) }]-4 \pi \tau_{2} \Delta_{b}^{(m)}+4 \pi \frac{\tau_{2}}{|\tau|^{2}} \Delta_{a}^{(m|\tau|)} .
\end{aligned}
$$

Further we again employ the Poisson resummation with respect to $p$ and then the integral part of the final expression in (A.3) becomes

$$
\begin{aligned}
& -\frac{1}{\pi|\tau|} \sum_{\hat{p}=-\infty}^{\infty} \sum_{\hat{n}=1}^{\infty} \int_{0}^{\infty} d s s^{-\frac{1}{2}} \exp \left(-\frac{s \hat{n}^{2}}{|\tau|^{2}}-\frac{\pi^{2} \tau_{2}^{2}}{s}\left(\frac{(\hat{p} \pm a)^{2}}{|\tau|^{2}}+m^{2}\right) \mp 2 \pi i b n \mp 2 \pi i(\hat{p} \pm a) \frac{\tau_{1} \hat{n}}{|\tau|^{2}}\right) \\
& =-\sum_{\hat{n}=1}^{\infty} \frac{1}{\hat{n}} \sum_{\hat{p}=-\infty}^{\infty} \exp \left(-2 \pi \hat{n} \tau_{2} \sqrt{\frac{(\hat{p} \pm a)^{2}}{|\tau|^{4}}+\frac{m^{2}}{|\tau|^{2}}} \mp 2 \pi i(\hat{p} \pm a) \frac{\tau_{1} \hat{n}}{|\tau|^{2}} \mp 2 \pi i b \hat{n}\right) .
\end{aligned}
$$

Putting this result (A.4) into (A.3) we can easily find the modular transformation of $Z_{a, b}^{(m)}(\tau, \bar{\tau})$ (2.12). 


\section{References}

[1] R. R. Metsaev, "Type IIB Green-Schwarz superstring in plane wave Ramond-Ramond background," Nucl. Phys. B 625 (2002) 70, hep-th/0112044; R. R. Metsaev and A. A. Tseytlin, "Exactly solvable model of superstring in plane wave Ramond-Ramond background," hepth/0202109.

[2] R. Penrose, "Any space-time has a plane wave as a limit," Differential geometry and relativity, Reidel, Dordrecht, 1976 (271).

[3] M. Blau, J. Figueroa-O'Farrill, C. Hull and G. Papadopoulos, "A new maximally supersymmetric background of IIB superstring theory," JHEP 0201 (2002) 047, hep-th/0110242; M. Blau, J. Figueroa-O'Farrill, C. Hull and G. Papadopoulos, "Penrose limits and maximal supersymmetry," Class. Quant. Grav. 19 (2002) L87, hep-th/0201081; M. Blau, J. Figueroa-O'Farrill and G. Papadopoulos, "Penrose limits, supergravity and brane dynamics," hep-th/0202111.

[4] D. Berenstein, J. Maldacena and H. Nastase, "Strings in flat space and pp waves from N = 4 super Yang Mills," JHEP 0204 (2002) 013, hep-th/0202021.

[5] M. B. Green, J. H. Schwarz and E. Witten, "Superstring Theory. Vol. 1," Cambridge, Uk: Univ. Pr. (1987).

[6] N. Itzhaki, I. R. Klebanov and S. Mukhi, "PP wave limit and enhanced supersymmetry in gauge theories," JHEP 0203 (2002) 048, hep-th/0202153.

[7] J. Gomis and H. Ooguri, "Penrose limit of $\mathrm{N}=1$ gauge theories," hep-th/0202157; L. A. Zayas and J. Sonnenschein, "On Penrose limits and gauge theories," JHEP 0205 (2002) 010, hep-th/0202186; U. Gursoy, C. Nunez and M. Schvellinger, "RG flows from Spin(7), CY 4-fold and HK manifolds to AdS, Penrose limits and pp waves," hep-th/0203124; C. h. Ahn, "More on Penrose limit of $\operatorname{AdS}(4) \times \mathrm{Q}^{* *}(1,1,1)$," hep-th/0205008: K. Oh and R. Tatar, "Orbifolds, Penrose limits and supersymmetry enhancement," hep-th/0205067; C. h. Ahn, "Comments on Penrose limit of AdS(4) x M**(1,1,1)," hep-th/0205109.

[8] J. G. Russo and A. A. Tseytlin, "On solvable models of type IIB superstring in NS-NS and R-R plane wave backgrounds," JHEP 0204 (2002) 021, hep-th/0202179.

[9] M. Hatsuda, K. Kamimura and M. Sakaguchi, "From super-AdS(5) x S**5 algebra to superpp-wave algebra," hep-th/0202190: M. Hatsuda, K. Kamimura and M. Sakaguchi, "SuperPP-wave algebra from super-AdS x S algebras in eleven-dimensions," hep-th/0204002; G. Arutyunov and E. Sokatchev, "Conformal fields in the pp-wave limit," hep-th/0205270.

[10] M. Billo and I. Pesando, "Boundary states for GS superstrings in an Hpp wave background," hep-th/0203028; C. S. Chu and P. M. Ho, "Noncommutative D-brane and open string in pp-wave background with B-field," hep-th/0203186; A. Dabholkar and S. Parvizi, "Dp branes in pp-wave background," hep-th/0203231; D. Berenstein, E. Gava, J. Maldacena, K. S. Narain and H. Nastase, "Open strings on plane waves and their Yang-Mills duals," hep-th/0203249; P. Lee and J. w. Park, "Open strings in PP-wave background from defect conformal field theory," hep-th/0203257; A. Kumar, R. R. Nayak and Sanjay, "D-brane solutions in pp-wave background," hep-th/0204025; D. s. Bak, "Supersymmetric branes in PP wave background," hep-th/0204033; K. Skenderis and M. Taylor, "Branes in AdS and pp-wave spacetimes," hep-th/0204054; V. Balasubramanian, M. Huang, T. S. Levi and A. Naqvi, "Open Strings from N=4 Super Yang-Mills," hep-th/0204196: H. Singh, "M5-branes with 3/8 supersymmetry in pp-wave background," hep-th/0205020; P. Bain, P. Meessen and M. Zamaklar, "Supergravity solutions for D-branes in Hpp-wave backgrounds," hep-th/0205106; M. Alishahiha and A. Kumar, "D-brane solutions from new 
isometries of pp-waves," hep-th/0205134; C. S. Chu, P. M. Ho and F. L. Lin, "Cubic string field theory in pp-wave background and background independent Moyal structure," hep-th/0205218; S. Seki, "D5-brane in Anti-de Sitter Space and Penrose Limit," hep-th/0205266; D. Mateos and S. Ng, "Penrose Limits of the Baryonic D5-brane," hepth/0205291: S. S. Pal, "Solution to Worldvolume Action of D3 brane in pp-wave Background," hep-th/0205303.

[11] M. Alishahiha and M. M. Sheikh-Jabbari, "The PP-wave limits of orbifolded AdS(5) x $\mathrm{S}^{* *} 5, "$ hep-th/0203018.

[12] N. w. Kim, A. Pankiewicz, S. J. Rey and S. Theisen, "Superstring on pp-wave orbifold from large-N quiver gauge theory," hep-th/0203080.

[13] T. Takayanagi and S. Terashima, "Strings on orbifolded pp-waves," hep-th/0203093.

[14] E. Floratos and A. Kehagias, "Penrose limits of orbifolds and orientifolds," hep-th/0203134.

[15] M. Cvetic, H. Lu and C. N. Pope, "Penrose limits, pp-waves and deformed M2-branes," hep-th/0203082; M. Cvetic, H. Lu and C. N. Pope, "M-theory pp-waves, Penrose limits and supernumerary supersymmetries," hep-th/0203229; J. P. Gauntlett and C. M. Hull, "pp-waves in 11-dimensions with extra supersymmetry," hep-th/0203255: H. Lu and J. F. Vazquez-Poritz, "Penrose limits of non-standard brane intersections," hep-th/0204001; R. Corrado, N. Halmagyi, K. D. Kennaway and N. P. Warner, "Penrose Limits of RG Fixed Points and PP-Waves with Background Fluxes," hep-th/0205314.

[16] J. Michelson, "(Twisted) toroidal compactification of pp-waves," hep-th/0203140.

[17] R. Gueven, "Randall-Sundrum zero mode as a Penrose limit," hep-th/0203153; M. Li, "Correspondence principle in a pp-wave background," hep-th/0205043; S. D. Mathur, A. Saxena and Y. K. Srivastava, "Scalar propagator in the pp-wave geometry obtained from AdS(5) x S**5," hep-th/0205136; G. Siopsis, "Holography in the Penrose limit of AdS space," hep-th/0205302.

[18] S. R. Das, C. Gomez and S. J. Rey, "Penrose limit, spontaneous symmetry breaking and holography in pp-wave background," hep-th/0203164; E. Kiritsis and B. Pioline, "Strings in homogeneous gravitational waves and null holography," hep-th/0204004; R. G. Leigh, K. Okuyama and M. Rozali, "PP-waves and holography," hep-th/0204026; Y. Imamura, "Large angular momentum closed strings colliding with D-branes," hep-th/0204200.

[19] N. Berkovits, "Conformal field theory for the superstring in a Ramond-Ramond plane wave background," JHEP 0204 (2002) 037, hep-th/0203248.

[20] S. S. Gubser, I. R. Klebanov and A. M. Polyakov, "A semi-classical limit of the gauge/string correspondence," hep-th/0204051; S. Frolov and A. A. Tseytlin, "Semiclassical quantization of rotating superstring in $\operatorname{AdS}(5) \times \mathrm{S}^{* *} 5$," hep-th/0204226; J. G. Russo, "Anomalous dimensions in gauge theories from rotating strings in $A d S_{5} \times S^{5}$," hep-th/0205244; A. Armoni, J. L. Barbon and A. C. Petkou, "Orbiting strings in AdS black holes and N=4 SYM at finite temperature," hep-th/0205280.

[21] M. Spradlin and A. Volovich, "Superstring Interactions in a pp-wave Background," hepth/0204146.

[22] S. Mukhi, M. Rangamani and E. Verlinde, "Strings from Quivers, Membranes from Moose," hep-th/0204147; M. Alishahiha and M. M. Sheikh-Jabbari, "Strings in PP-Waves and Worldsheet Deconstruction," hep-th/0204174.

[23] H. Takayanagi and T. Takayanagi, "Open strings in exactly solvable model of curved spacetime and PP-wave limit," JHEP 0205 (2002) 012, hep-th/0204234. 
[24] I. Bakas and K. Sfetsos, "PP-waves and logarithmic conformal field theories," hepth/0205006; A. Parnachev and D. A. Sahakyan, "Penrose limit and string quantization in AdS(3) x S**3," hep-th/0205015; V. E. Hubeny, M. Rangamani and E. Verlinde, "Penrose Limits and Non-local theories," hep-th/0205258.

[25] D. Berenstein and H. Nastase, "On lightcone string field theory from super Yang-Mills and holography," hep-th/0205048.

[26] C. Kristjansen, J. Plefka, G. W. Semenoff and M. Staudacher, "A new double-scaling limit of $\mathrm{N}=4$ super Yang-Mills theory and PP-wave strings," hep-th/0205033; D. J. Gross, A. Mikhailov and R. Roiban, "Operators with large $\mathrm{R}$ charge in $\mathrm{N}=4$ Yang-Mills theory," hep-th/0205066; N. R. Constable, D. Z. Freedman, M. Headrick, S. Minwalla, L. Motl, A. Postnikov and W. Skiba, "PP-wave string interactions from perturbative Yang-Mills theory," hep-th/0205089; R. Gopakumar, "String interactions in PP-waves," hep-th/0205174; Y. j. Kiem, Y. b. Kim, S. m. Lee and J. m. Park, "PP-wave/Yang-Mills Correspondence: An Explicit Check," hep-th/0205279; M. -x. Huang, "Three point functions of N=4 Super Yang Mills from light cone string field theory in pp-wave," hep-th/0205311.

[27] O. Bergman, M. R. Gaberdiel and M. B. Green, "D-brane interactions in type IIB planewave background," hep-th/0205183.

[28] K. Dasgupta, M. M. Sheikh-Jabbari and M. Van Raamsdonk, "Matrix perturbation theory for M-theory on a PP-wave," hep-th/0205185; G. Bonelli, "Matrix strings in pp-wave backgrounds from deformed super Yang-Mills theory," hep-th/0205213.

[29] Y. Hikida and Y. Sugawara, "Superstrings on PP-wave backgrounds and symmetric orbifolds," hep-th/0205200.

[30] F. Bigazzi, A. L. Cotrone, L. Girardello and A. Zaffaroni, "PP-wave and Nonsupersymmetric Gauge Theory," hep-th/0205296

[31] I. R. Klebanov and A. A. Tseytlin, "D-branes and dual gauge theories in type 0 strings," Nucl. Phys. B 546 (1999) 155, hep-th/9811035; I. R. Klebanov and A. A. Tseytlin, "A non-supersymmetric large N CFT from type 0 string theory," JHEP 9903 (1999) 015, hep-th/9901101.

[32] J. Polchinski, "String Theory. Vol. 1,2," Cambridge, UK: Univ. Pr. (1998).

[33] M. R. Douglas and G. W. Moore, "D-branes, Quivers, and ALE Instantons," hepth/9603167; D. Diaconescu, M. R. Douglas and J. Gomis, "Fractional branes and wrapped branes," JHEP9802 (1998) 013, hep-th/9712230.

[34] A. Adams, J. Polchinski and E. Silverstein, "Don't panic! Closed string tachyons in ALE space-times," JHEP 0110 (2001) 029, hep-th/0108075.

[35] T. Takayanagi and T. Uesugi, "Orbifolds as Melvin geometry," JHEP 0112 (2001) 004, hep-th/0110099.

[36] A. Dabholkar, "Strings on a cone and black hole entropy," Nucl. Phys. B 439 (1995) 650,hep-th/9408098; D. A. Lowe and A. Strominger, "Strings near a Rindler or black hole horizon," Phys. Rev. D 51 (1995) 1793, hep-th/9410215.

[37] N. Arkani-Hamed, A. G. Cohen and H. Georgi, "(De)constructing dimensions," Phys. Rev. Lett. 86 (2001) 4757, hep-th/0104005; N. Arkani-Hamed, A. G. Cohen, D. B. Kaplan, A. Karch and L. Motl, "Deconstructing $(2,0)$ and little string theories," hep-th/0110146.

[38] I. Rothstein and W. Skiba, "Mother moose: Generating extra dimensions from simple groups at large N," Phys. Rev. D 65 (2002) 065002, hep-th/0109175. 